\title{
Fall prevention interventions for older community-dwelling adults: systematic reviews on benefits, harms, and patient values and preferences
}

Jennifer Pillay ${ }^{1 *}$ (D), John J. Riva ${ }^{2}$, Laure A. Tessier ${ }^{3}$, Heather Colquhoun ${ }^{4}$, Eddy Lang ${ }^{5}$, Ainsley E. Moore ${ }^{2}$, Brett D. Thombs ${ }^{6}$, Brenda J. Wilson ${ }^{7}$, Amanda Tzenov ${ }^{8}$, Catherine Donnelly ${ }^{9}$, Marcel Émond ${ }^{10}$, Jayna Holroyd-Leduc ${ }^{11}$, Jamie Milligan ${ }^{12}$, Diana Keto-Lambert ${ }^{1}$, Sholeh Rahman', Ben Vandermeer ${ }^{1}$, Andrea C. Tricco ${ }^{13}$, Sharon E. Straus ${ }^{13}$, Sonia M. Thomas ${ }^{13}$, Bradley R. Mitchelmore ${ }^{3}$, Elizabeth Rolland-Harris ${ }^{3}$ and Lisa Hartling ${ }^{1}$

\begin{abstract}
Background: An estimated 20-30\% of community-dwelling Canadian adults aged 65 years or older experience one or more falls each year. Fall-related injuries are a leading cause of hospitalization and can lead to functional independence. Many fall prevention interventions, often based on modifiable risk factors, have been studied. Apart from the magnitude of the benefits and harms from different interventions, the preferences of older adults for different interventions as well as the relative importance they place on the different potential outcomes may influence recommendations by guideline panels. These reviews on benefits and harms of interventions, and on patient values and preferences, will inform the Canadian Task Force on Preventive Health Care to develop recommendations on fall prevention for primary care providers.
\end{abstract}

Methods: To review the benefits and harms of fall prevention interventions, we will update a previous systematic review of randomized controlled trials with adaptations to modify the classification of interventions and narrow the scope to community-dwelling older adults and primary-care relevant interventions. Four databases (MEDLINE, Embase, Cochrane Central Register of Controlled Trials, Ageline), reference lists, trial registries, and relevant websites will be searched, using limits for randomized trials and date (2016 onwards). We will classify interventions according to the Prevention of Falls Network Europe (ProFANE) Group's taxonomy. Outcomes include fallers, falls, injurious falls, fractures, hip fractures, institutionalization, health-related quality of life, functional status, and interventionrelated adverse effects. For studies not included in the previous review, screening, study selection, data extraction on outcomes, and risk of bias assessments will be independently undertaken by two reviewers with consensus used for final decisions. Where quantitative analysis is suitable, network or pairwise meta-analysis will be conducted using (Continued on next page)

\footnotetext{
*Correspondence: jpillay@ualberta.ca

'Alberta Research Centre for Health Evidence, University of Alberta, 1140587 Avenue, Edmonton, AB T6G 1C9, Canada

Full list of author information is available at the end of the article
}



(c) Crown 2021 Open Access This article is licensed under a Creative Commons Attribution 4.0 International License, which permits use, sharing, adaptation, distribution and reproduction in any medium or format, as long as you give appropriate credit to the original author(s) and the source, provide a link to the Creative Commons licence, and indicate if changes were made. The images or other third party material in this article are included in the article's Creative Commons licence, unless indicated otherwise in a credit line to the material. If material is not included in the article's Creative Commons licence and your intended use is not permitted by statutory regulation or exceeds the permitted use, you will need to obtain permission directly from the copyright holder. To view a copy of this licence, visit http://creativecommons.org/licenses/by/4.0/. The Creative Commons Public Domain Dedication waiver (http://creativecommons.org/publicdomain/zero/1.0/) applies to the data made available in this article, unless otherwise stated in a credit line to the data. 


\begin{abstract}
(Continued from previous page)
a frequentist approach in Stata. Assessment of the transitivity and coherence of the network meta-analyses will be undertaken. For the reviews on patient preferences and outcome valuation (relative importance of outcomes), we will perform de novo reviews with searches in three databases (MEDLINE, Psyclnfo, and CINAHL) and reference lists for cross-sectional, longitudinal quantitative, or qualitative studies published from 2000. Selection, data extraction, and risk of bias assessments suitable for each study design will be performed in duplicate. The analysis will be guided by a narrative synthesis approach, which may include meta-analysis for health-state utilities. We will use the CINeMa approach to a rate the certainty of the evidence for outcomes on intervention effects analyzed using network meta-analysis and the GRADE approach for all other outcomes.
\end{abstract}

Discussion: We will describe the flow of literature and characteristics of all studies and present results of all analyses and summary of finding tables. We will compare our findings to others and discuss the limitations of the reviews and the available literature.

Systematic review registration: This protocol has not been registered.

Keywords: Fall prevention, Systematic review, Patient values and preferences, Guideline, CINeMA, GRADE

\section{Background}

\section{Burden of falls}

An estimated $20-30 \%$ of older Canadian adults (> 65 years old) living in the community experience one or more falls each year [1]. In older adults, fall-related injuries (limiting normal activities) occur more frequently in females than in males (e.g., 67 vs. 46 per 1000 in 2010) and increase with age-rates for those $\geq 85$ years of age are about double for those 65 to 74 years of age [1].

Approximately $10 \%$ of falls among older adults result in a fracture $[2,3]$. The majority of cost, morbidity, and mortality generated by fall-related injuries are attributed to peripheral fractures, especially at the hip, rather than vertebral fractures or injuries to the soft tissues or organs $[1,4]$. Less serious injuries, such as bruising, lacerations, and sprains, can still lead to pain, reduced function, and substantial costs to the individual and healthcare system [5]. In Canada, the leading causes of fall-related injuries in older adults were walking ( $16 \%$ on snow or ice and $45 \%$ on other surfaces) and accidents going up or downstairs (13\% of injuries) [1]. The 2009/ 10 Canadian Community Health Survey found that the most common fall-related injury in older adults was a fracture (35\%) followed by sprains/strains (30\%) [1]. Emergency room visits following a fall are not limited to those experiencing a fracture; the survey also found that of older adults who reported seeking treatment related to a fall, most (67\%) went to an emergency room. Injuries resulting from a fall are a leading cause of hospitalizations among Canadian adults aged $>65$ years, and the length of hospitalization from fall-related injuries is on average 9 days longer (e.g., 21 vs. 12 days) than for all causes of hospitalization [1]. Based on data from Canadian acute care hospitals, $17 \%$ of older adults who were hospitalized for a fall were moved from living in the community to a continuing care facility [6]. Many older people also experience psychological difficulties after falls, including a fear of falling or loss of confidence, which may contribute to further falling [7]. Falls have been associated with reduced quality of life [8] and can lead to loss of function in activities of daily living (ADLs), thereby impacting independence and overall health outcomes [9].

\section{Risk factors and screening for risk}

Falls often are a result of a complex combination of risk factors, interacting to cause an inability to maintain or regain one's balance [1]. Risk factors may be classified as (i) biological, related to disease(s), and the natural aging process (e.g., balance and gait deficiency, acute or chronic health conditions, cognitive impairment, low vision); (ii) behavioral, such as the use of unsuitable/poorly maintained assistive devices including footwear and clothing, fear of falling, the use of certain medications (e.g., psychotropic, sedatives, hypnotics), and vitamin D intake to improve the function of the skeletal muscle $[10,11])$; (iii) social and economic (e.g., social isolation, poverty, poor access to healthcare); and (iv) environmental from factors in the community (e.g., building entrances, lack of handrails), living environment (e.g., type of furniture, home clutter), and/or related to weather and climate (e.g., icy surfaces) [1].

Screening patients for their risk for falls has been considered, with the aim of determining who may benefit the most from further assessment and/or an intervention to prevent falls. Many different methods for screening have been developed or considered, relying on single or multiple-item history questions, self-report measures/ questionnaires (e.g., Falls Efficacy Scale [12]), or performance-based measures (e.g., 30 Second Chair Stand [13], Berg Balance Scale [14], and Timed Up and Go [15]). However, no single screening method has demonstrated to be simple and highly accurate for predicting risk, intervention effects may not be modified by 
fall risk status, and we are not aware of any trials or systematic reviews on screening effectiveness. A 2018 systematic review of 26 assessment tools for falls risk in older adults found that no single tool showed sufficiently high predictive validity (e.g., sensitivities and area-underthe curves ranged from 63 to $76 \%$ and 0.76 to 0.81 , respectively, for those used in community-dwelling populations) to differentiate between people at high versus low risk of falls [16]. The authors concluded that clinicians should consider using at least two assessment tools together to better evaluate their patients' risk. Likewise, when evaluating the predictive ability of single and combined use of medical history questions, authors of another review concluded that no single question emerged as a powerful predictive tool, but that querying several factors together (i.e., fall history, difficulty with ADLs, the use of an ambulatory device, concern about falling, and use of psychoactive medication) could be useful (e.g., using a calculation of cumulative post-test probability to indicate that a patient's risk for falling would change from $30 \%$ without screening to $\geq 60 \%$ ) [17]. The time burden to adopt a multiple tool-based or question strategy may lead to unacceptably low uptake by primary care providers due to competing demands that occur in the care of older patients, often with multiple comorbidities to manage during a clinical encounter. Further, several reviews of fall prevention intervention studies have found no significant difference in effects for exercise, multiple component, or multifactorial interventions based on whether or not above-average or high-fall risk was used for study inclusion [18-20].

\section{Fall prevention interventions}

Many interventions for preventing falls have been studied. These are often based on known, modifiable risk factors for falling. Most fall prevention interventions can be classified according to the internationally accepted taxonomy developed by the Prevention of Falls Network Europe (ProFANE) Group [21]. A major feature of the taxonomy is the distinction between different categories and combinations of interventions. Interventions may comprise single-component interventions, involving one or a combination of two or more interventions (e.g., gait and balance training with strength/resistance exercises) from the same category (e.g., exercise), or interventions where more than one intervention from different categories are offered to everyone (multiple component interventions) or tailored based on an individual's risk assessment (multifactorial interventions). Single and multiple component interventions may include some form of fall risk assessment or be directed towards those with one or more particular risk factor(s), but they do not tailor the intervention components to each individual's risk as do multifactorial interventions. Some of the interventions within the taxonomy are more applicable than others to the general population of communitydwelling older adults and the practice of primary care (i.e., first-contact, accessible, continued, comprehensive and coordinated care [22]). These may be provided directly by primary care providers (e.g., vitamin D supplementation, nurse-led education on falls risk and prevention), by an inter-professional team of providers (e.g., exercise and cognitive-behavioral therapy), through referral to an allied health care provider (e.g., environmental/home hazard assessment), or in the community (e.g., patient-initiated attendance at Tai Chi classes). Other interventions, such as management of urinary incontinence or cataract surgery, target populations having a specific diagnosis or condition and, when given alone, are not considered to address the primary aim of fall prevention. Interventions may differ depending on the population (e.g., general vs. increased risk based on recruitment from emergency departments vs. frail) and delivery setting (e.g., community vs. nursing homes vs. hospitals). They may also focus on the primary prevention of a fall or secondary prevention of subsequent falls. Some interventions may be better considered "add-on" strategies, that are likely insufficient on their own to prevent falls, but rather used to enhance the uptake or implementation of a main intervention (e.g., patient appointment reminders, clinician training in exercise therapy, or fall risk assessment).

\section{Relevance of values and preferences}

Health-care decision-making is influenced by the health effects of interventions as well as people's values and preferences [23]. While acceptance rates across various types of fall prevention interventions, on average, appear quite high (e.g., approximately $70 \%$ of older adults agree to participate in studies regardless of eligibility [24]), there is evidence on stated preferences for different types and/or formats of interventions (e.g., [25-27]) that could inform decisions about which interventions to recommend in general and to specific populations. Moreover, preferences for or against an intervention are viewed as a consequence of the relative importance people place on the expected or experienced health outcomes it incurs [28]. When considering multiple different types of interventions where the impact on different outcomes may vary between types, the relative importance placed by patients on the different potential outcomes may influence which interventions are considered more or less effective.

\section{Aims and rationale for reviews}

The findings of three systematic reviews will be used by the Canadian Task Force on Preventive Health Caresupplemented by input from patient and organizational 
stakeholder consultations and by other sources of information on feasibility, acceptability, costs/resources, and equity - to make recommendations for primary care providers on fall prevention interventions. The following key questions (KQs) will be answered.

KQ1: What are the benefits and harms of interventions compared with usual care to prevent falls in community-dwelling adults aged 65 and older? KQ2: How do community-dwelling adults aged 65 and older weigh the potential benefits and harms of interventions to prevent falls?

KQ3: What are the preferences of community-dwelling adults aged 65 and older regarding different interventions demonstrated to prevent falls?

A comprehensive search for systematic reviews related to our KQs published between 2014 and 2019 found that while many systematic reviews $(>80)$ exist, most have focused on specific types of interventions (e.g., exercise) and/or specific populations (e.g., cognitively impaired). Although some recent reviews could be considered as closely meeting our aim for KQ1 on benefits and harms of various interventions [18, 29-31], no review matches this review's scope fully in terms of their population, interventions, comparators, outcomes, timing, and setting (i.e., PICOTS). To avoid duplication of effort and build on others' work, we will rely on a review with the broadest scope/PICOTS [29] for identification and data extraction of studies related to KQ1 on benefits and harms. We will update the literature base and make adaptations where suitable to meet the narrower scope determined by the Task Force (e.g., exclude interventions delivered exclusively in hospital or nursing home settings). We did not identify an existing review fully answering KQ2 on the relative importance of outcomes or KQ3 on preferences for different interventions, respectively, and will therefore conduct de novo reviews for these two questions.

\section{Methods}

The Evidence Review and Synthesis Centre at the University of Alberta's Alberta Research Centre for Health Evidence will complete the reviews (JP, DK-L, BV, SR, LH). The reviews will be developed, conducted, and prepared according to the Task Force methods [32], using methods guided by the Cochrane Handbook [33] and Grading of Recommendations Assessment, Development and Evaluation (GRADE) working group [23, 34, 35]. This protocol follows reporting standards [36]. The review for KQ1 on the benefits and harms of fall prevention interventions will be conducted in collaboration with the authors of the review which is being adapted (ACT, SES, SMT) [29]. The protocol was reviewed by peer-reviewers and organizational stakeholders $(n=9)$. This final version of the protocol has been approved by the entire Task Force.

A working group of Task Force members (JJR, HC, EL, AEM, BDT, BJW, AT) and content experts (CD, ME, $\mathrm{JH}-\mathrm{L}, \mathrm{JM}$ ) was formed for development of KQs and PICOTS. Task Force members chose and rated outcomes in terms of their importance for creating a recommendation, according to methods of GRADE [35]. Outcome ratings were finalized after input from an outcome rating exercise and focus groups conducted with a sample of older adults in Canada, by an independent group, led by SES, with expertise in knowledge translation from St. Michael's Hospital in Toronto, Ontario. Eight outcomes were considered critical for decision making (i.e., rated 7 or above on a scale of 1-9) by the Task Force: number of fallers, number of falls, number of injurious falls, number of fractures, number of hip fractures, residential status/institutionalization, health-related quality of life, and functional status. Intervention-related adverse effects (AEs; any or serious) were rated as important (i.e., rated 4-6) and included.

The Science Team of the Global Health and Guidelines Division at the Public Health Agency of Canada (PHAC) (LAT, BM, ERH) provided assistance and input on Task Force methodological considerations during the development of the protocol.

\section{Eligibility criteria}

Tables 1,3 , and 4 outline each KQ's study eligibility criteria (i.e., PICOTS). Table 2 is specific to the components of fall prevention interventions, and study comparators, of interest.

The main population of interest for all $\mathrm{KQs}$ is adults aged 65 or older living in the community, that is, at home or in independent living/retirement facilities where no or minimal assistance is provided. We will include studies only recruiting people who have never fallen as well as those that include people who have a history of falls. For KQ2, when looking at the valuation/ importance of the outcomes of fractures and transfer to residential status/institutionalization, we will also include studies of populations newly admitted to residential care/nursing homes. We will exclude studies with recruitment based exclusively on one or more specific medical diagnoses (e.g., stroke, Parkinson's disease), because these populations are expected to require fall prevention interventions and management/usual care that are substantially different from those applicable to the general population of community-dwelling adults. For KQ2 and 3, studies may include family members or caregivers who participate on behalf of people with cognitive impairment or otherwise unable to understand the study procedures. 
Table 1 Eligibility criteria for key question 1

\begin{tabular}{ll}
\hline Inclusion criteria \\
\hline Population & Adults living in the community, aged 65 or older. \\
Community living consists of living at home or in independent \\
living/retirement facilities where no or minimal assistance (e.g., \\
help with one activity of daily living [ADL; e.g. bathing] or \\
Instrumental ADL, e.g. cooking) is provided \\
Studies recruiting adults living in the community under the \\
age of 65 may be included if $\geq 80 \%$ of the participants are \\
aged 65 or older. If the proportion of the participants aged 65 \\
or older is not available, studies may be included if the \\
participants' mean age minus one standard deviation is equal \\
to or greater than 65.
\end{tabular}

Interventions

Comparator

Outcomes

Timing

Delivery Setting Any relevant to primary care (primary care, community [home or other]).

Interventions can be initiated in the emergency department but cannot be entirely delivered in the emergency department.

- Usual care (e.g., no additional care focusing on falls; may include wait-list, attention control, pamphlet or generic health education, placebo) falls risk, social visits)

- Another intervention to prevent falls; for critical outcomes where NMA is conducted only, and if classified differently according to ProFANE taxonomy and our coding

\section{Critical}

- Falls (i.e., total number of falls per unit of person-time)

- Fallers (i.e., number of people falling one or more times during follow-up)

- Injurious falls (one used per study using a hierarchy: falls leading to hospitalization, falls requiring emergency department visit, falls requiring physician visit, any injurious fall); preferentially the number of people with one or more injurious falls, but will include total number if necessary

- Fractures (only fall-related, if reported; preferentially the number of people with one or more fractures, but will include total number if necessary)

- Hip fractures (only fall-related, if reported)

- Residential status/institutionalization (number of people newly admitted to residential care)

- Health-related quality of life (validated scales, e.g., SF-12 and/ or SF-36 Physical and Mental Components, EQ 5D VAS, EuroQol EQ-5D)

- Functional status: (i) validated scales including activities of number of people with new/increased need for homecare assistance; (iii) other validated scales will be considered

\section{Important}

- Intervention-related adverse effects (AEs) as defined by study (people experiencing one or more AEs; individual serious AEs)

Follow-up duration: $\geq 3$ months after randomization

\section{Exclusion criteria}

Studies with recruitment based exclusively on one or more

specific diagnoses. Excluded populations include, but are not limited to:

- Stroke

- Parkinson's (neurodegenerative conditions)

- Severe dementia (will include if all mild-to-moderate

cognitive impairment)

- Long-term care facilities

- Housebound

- Severe frailty (with protocol for addressing falls or for falls risk assessment in place)

- Impaired balance (severe)

- Community-dwelling and receiving long-term, intensive

nursing care

- Visual impairment (severe)

- Hospitalized patients

- Acute fracture

- Confirmed vitamin D deficiency

Interventions that cannot feasibly or readily be delivered or referred to by a wide variety of primary care providers (see exclusions in Table 2).

Interventions that are not directly focused on the cascade of falls (i.e., falls prevention must be primary aim of intervention). Single interventions that are exclusively screening/assessment tools and/or quality improvement ("add-on") strategies.

- Non- or minimally active intervention (e.g., brief pamphlet on daily living and instrumental ADLs [composite scores only], (ii)
Settings not relevant to primary care and targeting general community-dwelling population (e.g., workplaces, inpatient settings, specialist settings, interventions entirely delivered in the emergency departments, nursing/long-term care homes). 
Table 1 Eligibility criteria for key question 1 (Continued)

\begin{tabular}{lll}
\hline & Inclusion criteria & Exclusion criteria \\
\hline Study design & $\begin{array}{l}\text { Randomized controlled trials (all designs including parallel, } \\
\text { cluster, crossover, multifactorial) }\end{array}$ & $\begin{array}{l}\text { - Editorials } \\
\text { - Commentaries }\end{array}$ \\
& & $\begin{array}{l}\text { Studies only published/available as conference proceedings, } \\
\text { letters, or other gray literature (e.g., government reports), } \\
\text { unless information on study design (e.g., eligibility criteria, } \\
\text { participant characteristics, intervention characteristics) is } \\
\text { described sufficiently and results are confirmed as final } \\
\text { (accessible online or via author contact) }\end{array}$ \\
$\begin{array}{l}\text { Language of } \\
\text { full text }\end{array}$ & English or French & \\
$\begin{array}{l}\text { Dates of } \\
\text { publication }\end{array}$ & Any & \\
\hline
\end{tabular}

For KQ1 on benefits and harms, we will include studies with at least one eligible single intervention as described in Table 2, of those chosen by the Task Force working group to reflect interventions having a primary aim to prevent falls in a broad population of community-dwelling older adults, and delivered in, or referable from, a primary care setting. We will exclude interventions that are solely used for screening or assessment, or as "add ons" to improve the uptake or implementation of interventions targeted at preventing falls but not proposed to reduce falls themselves. Participants can be recruited in hospitals, but the intervention must be primarily delivered outpatient in primary care or the community.

The main KQ1 comparator is usual care (UC), which is considered the medical and health care received by the target population within primary care that does not include any specific intervention to reduce falls. We will also include studies with a control having a non/minimally active intervention such as a pamphlet on falls risk or social engagement activities. We will seek clinical input in cases where there is uncertainty about whether the UC (as described by authors) is applicable to the general population of interest; if not applicable (e.g., comprehensive geriatric assessment is provided to all patients), the study will be excluded. Although the main interest of the Task Force is the effects of interventions versus UC, rather than the relative effects between different types of interventions, for critical outcomes, we will include head-to-head trials of different interventions and conduct network meta-analysis (NMA) to maximize the amount of data used and to generate estimates of the effects versus UC for those interventions that have not been (or have been minimally) studied in direct comparison with UC [37-39]. Studies that only compare different interventions that are both defined within one single intervention of our taxonomy (Table 2; e.g., different doses of vitamin $\mathrm{D}$ or intensities of strength training) will be excluded. Final inclusion of head-to-head comparisons will be based on the intervention (node) configurations in the final NMAs (see Data Synthesis for Key Question 1). For outcomes for which we do not undertake NMA, we will define the interventions as per the nodes used in the NMAs and only include studies using comparisons with UC or non-/minimally active interventions.

We will include randomized controlled trials (RCTs), of any design, with at least 3 months of follow-up after randomization to adequately capture the potential effects on the outcomes. Apart from English language reports, we will include those reported in French, as the Task Force considers reports published in both official languages in Canada (English and French). Literature suggests that language restrictions in systematic reviews on conventional medicine topics do not appear to bias results from meta-analyses [40, 41]. No restrictions will be placed on publication status, date, country, or risk of bias.

For KQs 2 and 3, the effects of the interventions are not of interest but rather the valuation/relative importance of the critical outcomes (KQ2) and the preferences of older adults for different interventions or intervention attributes (KQ3). The eligibility criteria for the studies in KQ2 align with those described by the GRADE working group [23, 28, 34]. For KQ2, we will prefer studies comparing two or more of the relevant outcomes (e.g., falls versus fractures) and/or with a comparison with a healthy population; studies without these will be considered if evidence is lacking on the importance of one or more outcomes. KQ3 will be conducted after KQ1, because we will only examine the preferences between different interventions, or between different attributes of interventions, that are shown to be effective by the KQ1 analysis. The attributes of interest will also be decided after completion of KQ1, but prior to study selection for KQ3. We will use a hierarchy of study designs for KQs 2 and 3 , in order to prioritize the most informative study designs for each KQ (see Tables 3 and 4). Qualitative studies would be very informative if the KQs were exploring reasoning (e.g., beliefs, barriers, expectations) 
Table 2 Modified ProFANE taxonomy [21] of interventions for inclusion and description of add-on strategies and comparators

\section{Category of intervention $^{a}$}

Exercise

Medication (drug provision)

Medication (review \& modification)

Nutrition therapy

Psychological

Environment/Assistive Technology (furnishings and adaptations to homes and other premises/direct action)

Environment/Assistive Technology (aids for personal mobility and protection)

Environment/Assistive Technology (aids for communication and signaling)

Environment/Assistive Technology (aids for communication and signaling)

Knowledge/education interventions

\section{Category of intervention add-on strategy ${ }^{b}$}

Social environment (clinic quality improvement)

Social environment (patient quality improvement)

\section{Control groups ${ }^{c}$}

Usual care (UC)

Non- or minimally active intervention (information)

\section{Single interventions within category ${ }^{\mathrm{b}}$}

Gait, balance, coordination, and functional training; strength/resistance exercises; flexibility exercises; 3D training (e.g., Tai Chi, dance, yoga); general physical activity; endurance training; others (e.g., exergame, aquatic); mixed exercises (i.e., 2 or more exercise components)

Vitamin D (+/-calcium) supplementation; sunlight interventions; excluding calcium alone, anti-osteoporosis medications or others used for specific conditions where fall prevention is secondary aim (e.g., diabetes medication, urinary antispasmodics)

Medication withdrawal, dose reduction or increase, substitution (may be delivered directly to patient or focus on provider education)

Dietary counselling; excluding single dietary supplements and fluid therapy

Cognitive (behavioral) interventions

Relocation, entrances, flooring, lighting, installation of grab bars in bathrooms, handrails for stairs, others

Walking aids, clothes, orthotics, or anti-slip devices for shoes; excluding protective aids to reduce fractures from falls (e.g., hip protectors) and comprehensive podiatry assessment

Alarm systems to prevent falls; excluding alarms to signal a fall, hearing aids, or optical aids unless part of a multiple component intervention

Vision assessment and treatment

Written material, videos, and lectures about reducing falls; excluding pamphlets

\section{Interventions within category}

Staff ratio, staff training, service model change, clinician reminders, audit and feedback, case management, referral (not for falls risk assessment or interventions which is captured by delivery variable); training and education to deliver the main interventions will not be counted (e.g., training of staff to deliver CBT or medication review)

Telephone support or reminders about appointments or aspects of care, caregiver training, homecare services, promotion of self-management (e.g., goal setting, action planning)

\section{Examples}

May include wait-list control, placebo (for vitamin D interventions), or session or pamphlet on general health or active living; excluding studies where UC involves as sessments (e.g., comprehensive geriatric) or interventions (potentially reducing falls) that are provided to all participants and not considered UC for the general community-dwelling population $\geq 65$ years of age ${ }^{d}$

UC as well as basic assessment related to falls risk factors without follow-up; brief pamphlet on falls risk or session on gentle exercises

Non- or minimally active intervention (social engagement)

UC as well as social visits/engagement, including group sessions

Abbreviations: $C B T$ cognitive behavioral therapy, UC usual care

a Interventions will also be categorized as single-component (e.g., one or more single interventions from a single category), multiple component (more than one single intervention from different categories offered to all people), and multifactorial (one or more single interventions are offered from different categories, based on an individual risk assessment). Multifactorial interventions will be assumed to include an assessment involving multiple falls risk factors, whereas other interventions will also be assumed to include some form of assessment (e.g., gait, medication review, dietary assessment)

${ }^{b}$ Excluded interventions and add-on quality improvement strategies cannot be the only intervention provided, but may be an included component of a multiple component or multifactorial intervention. Single interventions not specified in the table will be considered

${ }^{\mathrm{C}}$ All three possible control groups will be considered for the analysis; the forest plots will then be visually inspected to determine the degree of similarity between theses nodes, and they may be joined into 1 or 2 nodes thereafter

${ }^{\mathrm{d}}$ We will seek clinical input from the WG and clinical/topic experts before excluding any studies that may have UC that is not relevant/generalizable to the target population

behind the preferences, but the most relevant evidence on preferences as specified for these KQs is quantitative in nature. Studies reported in English or French will be sought. No restrictions will be placed on publication status, country, or risk of bias. We will limit inclusion to studies published on or after 2000 because it is expected that people's preferences change over time and because we expect a large proportion (> 90\%) of studies on fall prevention interventions to be conducted after this date [29].

\section{Searching the literature}

For KQ1 on benefits and harms, we will locate full texts of all studies included in the previous review [29]. 
Further, a librarian will update this review's peerreviewed searches (Additional file 1 contains the search for MEDLINE) from January 1, 2016, in Ovid MEDLINE (1946-), Ovid Embase (1996-), Wiley Cochrane Central Register of Controlled Trials (inception-), and Ageline. The search contains Medical Subject Heading terms and key words combining the concepts of falls/fallers, adults, and randomized controlled trials. Reference lists of all new trials and recent (2018 onwards) systematic reviews will be hand-searched by one reviewer. We will also search the World Health Organization Clinical Trials Search Portal (http://apps.who.int/trialsearch/), which searches multiple trial registries, and ask our clinical experts to provide us with a list of four to five organizational websites to search for conference abstracts and/or reports of research (2018 onwards). Where studies are only reported in conference abstracts or trial registries, first authors will be contacted by email (with two reminders over 1 month) to obtain full study reports and/or additional study or outcome data. If not received, these studies will be excluded with the reason documented.

A search for patient values and preferences (covering both KQs 2 and 3) has been developed combining Medical Subject Heading terms and key words for falls, fractures, and transition to residential care with those for patient preferences, quality of life, various preferencebased instrument/methodology terms (e.g., EQ-5D, conjoint analysis), decision making, attitudes, and acceptability (Additional file 1). This search has been peerreviewed by another librarian using the PRESS 2015 checklist [42]. For this KQ, we will search Ovid MEDL INE (1946-), Ovid PsycInfo (1987-), and CINAHL via EBSCOhost (1937-) databases and hand-search reference lists of included studies and of relevant systematic reviews.

We will export the results of database searches to an EndNote Library (version X7, Clarivate Analytics, Philadelphia, US, 2018) for record-keeping and will remove duplicates. We will document our supplementary search process, for any study not originating from the database searches, and enter these studies into EndNote individually. We will update electronic database searches for all KQs approximately 4 to 5 months prior to publication of the Task Force guideline. Results of new studies will be reported and, if considered to potentially impact conclusions and feasible, the relevant analyses will be rerun.

\section{Selection of studies}

Records retrieved from the database searches will be uploaded to DistillerSR (Evidence Partners Inc., Ottawa, Canada) for screening. For all citations retrieved from the database searches, two reviewers will independently screen all titles and abstracts using broad inclusion criteria. Full texts of any citation from the search considered potentially relevant by either reviewer will be retrieved. One exception is for the study designs in KQ2 on values and preferences that are lowest in our hierarchy (i.e., surveys, qualitative studies), where the full texts will only be reviewed if the other designs offer very low certainty evidence and we proceed to these designs. Two reviewers will independently review all full texts (including the studies from the previous review [29]) against a structured eligibility form, and a consensus process will be used for any full text not included by both reviewers. If necessary, a third reviewer with methods or clinical expertise and/or author contact will be used to arbitrate decisions. The screening and fulltext forms will be pilot-tested with a sample of at least 100 abstracts and 20 full texts, respectively, until the agreement is high (> 95\%). Screening studies located from reference lists, trial registries, and websites will be conducted by one experienced reviewer, with two reviewers reviewing full texts. Some exclusions are expected to occur after the final groupings/nodes of interventions is conducted (see below), should the study have no comparison between two different groups used for analysis. We will document the flow of records through the selection process, with reasons provided for all full-text exclusions, and present these in a PRISMA flow diagram [43] and appended excluded studies list.

\section{Data extraction}

We will rely on data extraction from the previous review team [29], as able and suitable. Because we are modifying the coding of interventions and adding an outcome of functional status, some data will be required to be extracted anew from the studies included in this review. For this data and for all data from new studies, one reviewer will extract data and another will verify all data for accuracy and completeness. We will adapt the data extraction form and related instructions used by the other review team, as necessary, and provide training for all reviewers involved in extraction. The data extraction form will be piloted with a sample of at least 10 studies, until agreement on all elements is high (>95\%).

Sufficient data will be collected to allow examination of the homogeneity and similarity assumptions for metaanalysis, and for assessment of the risk of bias, as described in the sections below. The main data items include the study characteristics (i.e., year and country of conduct, sample size enrolled, setting of recruitment [hospital vs. other], trial design); intervention(s) components (coded via Table 2), duration (total duration in weeks), dose (number of sessions/hours), assessment and delivery personnel (e.g., primary care provider or team vs. other); description of UC or other control (see 
Table 3 Eligibility criteria for key question 2

\begin{tabular}{|c|c|c|}
\hline Criteria & Inclusion & Exclusion \\
\hline Population & $\begin{array}{l}\text { Adults living in the community, aged } 65 \text { or older; when } \\
\text { looking at importance of outcome of residential status, } \\
\text { population may be awaiting or newly admitted to } \\
\text { residential care } \\
\text { Studies recruiting adults }<65 \text { years will be included if } \\
\geq 80 \% \text { of the participants are aged } 65 \text { or older, if } \\
\text { participants' mean age minus one standard deviation is } \geq \\
65 \text { years, or if results are provided for those } \geq 65 \text { years. } \\
\text { Family members or caregivers may serve as participants on } \\
\text { behalf of an older adult with cognitive impairment or } \\
\text { otherwise unable to understand the study procedures. }\end{array}$ & $\begin{array}{l}\text { Studies with recruitment based exclusively on one or more } \\
\text { specific diagnoses. Excluded populations include, but are } \\
\text { not limited to: } \\
\text { - Stroke } \\
\text { - Parkinson's (neurodegenerative conditions) } \\
\text { - Severe dementia } \\
\text { - Long-term care facilities (unless newly admitted) } \\
\text { - Housebound } \\
\text { - Severe frailty (with protocol for addressing falls or for falls } \\
\text { risk assessment in place) } \\
\text { - Impaired balance (severe) } \\
\text { - Community-dwelling and receiving long-term, intensive } \\
\text { nursing care (unless newly acquired need) } \\
\text { - Visual impairment (severe) } \\
\text { - Hospitalized patients (unless with acute fracture or injury } \\
\text { - Confirmed vitamin D deficiency }\end{array}$ \\
\hline
\end{tabular}

Exposure(s) - Experience with critical outcome(s) of interest, or - Exposure to clinical scenario(s) or information about potential critical outcome(s) and/or estimate(s) of effect on outcomes from falls prevention interventions, or

- No experience or exposure to information about critical outcomes, but authors are soliciting probability trade-offs (e.g., number of adverse events from interventions to make one fewer fall worthwhile) or ratings of different potential critical outcomes

Focus of study is on consideration of possible, or assessment of experienced, outcomes related to falls prevention that are considered critical by the Canadian Task Force on Preventive Health Care (see outcomes Table 1). For fractures, the main three "sub-outcomes" considered for this KQ will be "any fracture attributed to a fall", "any fracture" and "a single hip fracture".

Comparison(s) a) Experience or exposure to scenarios or information about a different critical outcome (e.g., falls vs. any fracture, hip vs. "any fracture")

b) Healthy state without critical outcome (for utility studies only)

c) No comparison (for utility studies only, if information from comparisons a or b are not available for a particular outcome)

Outcomes a) Utility values/weights for the potential outcomes/health states

b) Non-utility, quantitative information about relative importance of different outcomes, e.g., rating scales using ordinal or interval variables, ranking; preference for or against interventions [attendance, intentions, or acceptance] or preferred type of intervention based on different outcome risk descriptions, strength of associations between outcome ratings and behaviors or intentions for falls prevention interventions

c) Qualitative information indicating relative importance between outcomes

Data must relate to the outcomes considered critical to the Task Force (Table 1); for studies measuring the health state utility of a fracture or those residing in residential homes/ facilities, attribution to a fall will be prioritized, as possible Outcome groupings (a) to (c) above will be included in a hierarchical manner

Timing

Follow-up duration: any or none

Setting Any

Study Design and Publication Status

Any cross-sectional or longitudinal quantitative or qualitative study design using the methods described below:

Methods:
- Commentaries, opinion, editorials, case reports, and reviews

- Studies only published/available as conference proceedings or other grey literature (e.g., government 
Table 3 Eligibility criteria for key question 2 (Continued)

\begin{tabular}{|c|c|c|}
\hline Criteria & Inclusion & Exclusion \\
\hline & $\begin{array}{l}\text { a) Utility values/weights for health states measured directly } \\
\text { using time trade-off*, standard gamble**, visual analogue } \\
\text { scales, conjoint analysis with choice experiments or } \\
\text { probability trade-offs } \\
\text { b) Utility values/weights measured or estimated indirectly, } \\
\text { e.g., a person's health status is elicited along several } \\
\text { dimensions using a questionnaire (e.g., EuroQol-5D), then a } \\
\text { preference for that particular health state is derived, based } \\
\text { on values obtained from previous populations } \\
\text { c) Surveys or questionnaires with questions providing non- } \\
\text { utility, quantitative information about relative importance } \\
\text { of different outcomes; may be investigating decision aids } \\
\text { d) Qualitative studies providing information indicating } \\
\text { relative importance between benefits and harms } \\
\text { Study design groupings (c) and (d) will be included only if } \\
\text { insufficient data is available from (a) and (b) }\end{array}$ & $\begin{array}{l}\text { reports), unless information on study design (e.g., } \\
\text { eligibility criteria, participant characteristics, presentation } \\
\text { of scenarios) is available (accessible online or via author } \\
\text { contact) and sufficient to assess methodological quality. }\end{array}$ \\
\hline Language & English or French & \\
\hline Publication date & 2000-present & \\
\hline
\end{tabular}

Table 2); participant characteristics (sex, age, proportion with previous falls); and outcome tools, ascertainment, and result data (with sample size) at longest follow-up. Although not a focus for the analysis, studies with individuals or populations that may require equity (e.g., Indigenous peoples, newcomers to Canada, low income) [44] considerations by the Task Force will be noted and the applicability of the interventions to these populations will be assessed.

Table 1 contains our outcome definitions. Falls will often be defined as "an unexpected event in which the participant comes to rest on the ground, floor, or lower level" [45] although we will not exclude studies not using this or another definition. Fall-related injuries can be defined in various ways, focusing on symptoms (e.g., limiting one's normal activities, with or without fracture) and/or resource use (e.g., requiring attendance at the emergency department) [46]. To this end, if a study reports on various related fall-injury outomes, one will be extracted per study using a hierarchy based on assumed severity: falls leading to hospitalization, falls requiring emergency department visit, falls requiring physician visit, or any injurious fall. Of note, the previous review team allowed for data on falls to be included for their outcome of fallers, if the number of fallers was not reported and the number of falls was smaller than the study population. We are keeping these separate because rates of falls may be more sensitive to change than the proportion of fallers [20], and other reviews have found a difference in the effects between falls and number of fallers from falls prevention interventions [18-20]. For the outcomes of injurious falls, fractures, and hip fractures, we will rely on the number of people having one or more event but will include data on the number of events when necessary and assume that a participant would only have one event during follow-up. For the falls outcome, we will use raw data on incidence rates (number of falls per person-year) in each group where available; otherwise, we will calculate incidence rates or use the reported rate ratio $(\mathrm{RaR})$. For the other outcomes, we will extract the crude data on the number of people with the event and the sample size, unless only the risk ratio (RR) or odds ratio (OR) between study groups is reported. If studies report both adjusted and unadjusted ratios, we will use the unadjusted estimate unless the adjustment is for clustering. We will convert RRs to ORs for analysis.

We will record outcome data using an intention-totreat approach, where possible; if not possible, for instance when only relative effects/ratios between groups are reported instead of raw counts and intention-to-treat not used, we will rely on results from last-observedcarry-forward or, if necessary, per protocol/completer approaches.

When two or more interventions in a three- or fourarm trial are classified as having the same intervention as per our classification (e.g., different intensities of a strength training intervention), we will combine the results from the two interventions [33], to avoid loss of information.

For continuous outcomes measures, we will extract (by arm) the mean baseline and endpoint or change scores, standard deviations (SDs) or other measures of variability, and the number analyzed. If necessary, we will approximate means from medians. If SDs are not given, they will be computed or, if necessary estimated 
Table 4 Eligibility criteria for key question 3

\begin{tabular}{lll}
\hline Criteria & Inclusion & Exclusion \\
\hline Population & Adults living in the community, aged 65 or older & Studies with recruitment based exclusively on one or \\
Studies recruiting adults $<65$ years will be included if $\geq 80 \%$ & more specific diagnoses. Excluded populations include, \\
of the participants are aged 65 or older, if participants' mean & but are not limited to: \\
age minus one standard deviation is $\geq 65$ years, or if results & - Stroke & - Parkinson's (neurodegenerative conditions) \\
are provided for those $\geq 65$ years. & - Severe dementia \\
Family members or caregivers may serve as participants on & - Long-term care facilities \\
behalf of an older adult with cognitive impairment or & - Housebound \\
otherwise unable to understand the study procedures. & - Severe frailty (with protocol for addressing falls or for falls \\
& risk assessment in place) \\
& - Impaired balance (severe) \\
& - Community-dwelling and receiving long-term, intensive \\
& nursing care \\
& - Visual impairment (severe) \\
& - Hospitalized patients \\
& - Acute fracture \\
& - Confirmed vitamin D deficiency
\end{tabular}

Exposure(s) $\quad$ Experience with fall prevention interventions, or

- Exposure to information about different types and/or attributes of falls prevention interventions (e.g., mode, duration, setting, delivery providers, type of intervention): may include information about potential critical outcomes and/or estimates of effect on outcomes from falls prevention interventions, or

- No experience or exposure to information about interventions, but authors are soliciting information about preferred intervention attributes

Study must relate to types of interventions shown to be effective for at least one critical outcome, from analysis of KQ1. Studies may focus on different attribute(s) of effective interventions, particularly if shown in KQ1 to possibly moderate effects (i.e., specific attributes of interest will be determined based on findings from KQ1)

Comparison(s) $\quad$ a) Experience with different type of intervention, or b) Information about a different type of intervention, in terms of its components and/or attributes, or c) No comparison (in studies focusing on attributes within one type of intervention)

Outcomes

a) Quantitative data about preferences for intervention types or attributes from stated-preference valuation studies (e.g., willingness to pay or accept, preference weights/utility scores, odds ratios, coefficients)

b) Quantitative data from non-utility methods, about the relative importance of different intervention attributes (e.g., proportion preferring one type of intervention or attribute, intentions to participate, ranking or ratings of different interventions) c) Qualitative information indicating relative importance between different interventions

Outcome groupings (a) to (c) above will be included in a hierarchical manner

Timing

Follow-up duration: any or none

Setting

Any

Study Design and

Any cross-sectional or longitudinal quantitative or qualitative study design evaluating preferences between two or more intervention types or attributes of interest, using the methods described below: Methods:

a) Contingent valuation or choice experiments (e.g., discrete choice, contingent ranking, or best-worst scaling choice experiment)

b) Surveys/questionnaires or studies evaluating decision aids c) Qualitative studies providing information indicating relative importance between benefits and harms

Study design groupings (a) to (c) will be included in a hierarchical manner

Language

English or French

- Studies only published/available as conference proceedings or other grey literature (e.g., government reports), unless information on study design (e.g., eligibility criteria, participant characteristics, presentation of scenarios) is available (accessible online or via author contact) and sufficient to assess methodological quality.

- Commentaries, opinion, editorials, case reports, and reviews 
using established imputation methods [33]. When computing SDs for change from baseline values, we will assume a correlation of 0.5 , unless other information is present in the study that allows us to compute it more precisely [47]. We will use available software (i.e., Plot Digitizer, http://plotdigitizer.sourceforge.net/) to estimate effects from figures if no numerical values are provided.

We will use an intraclass correlation coefficient (ICC) of 0.01 [48], to adjust findings in cluster-design RCTs that have not done this. We will not adjust studies that randomize by household, considering the likelihood of the clustering effect to be very small [19]. If cross-over trials are included, we will limit the data extraction to the first period of the study, because of the potential for carry-over effects from the nature of fall prevention interventions, and treat the trial as if it used a parallelgroup design; the possible unit-of-analysis error introduced is recognized to provide a conservative estimate of the trial effects [33].

For KQs 2 and 3 on patient values and preferences, we will collect data on the population (as per KQ1) as well as exposure to any of the related outcomes and/or to fall prevention interventions. We will extract details about any instrument used, including development and composition of scenarios of health states, choice tasks including definitions of all attributes, or survey questions. Any details provided to participants about the potential benefits and harms of fall prevention interventions will be extracted. Where studies provide results (e.g., health utility values) for more than one type of falls (e.g., people falling once, twice, and more) or fracture outcome (e.g., wrist, tibia, distal femur), we will extract the findings as a range. If including qualitative studies, any relevant section of the results section will be pasted into a Microsoft Excel spreadsheet for further analysis.

We will contact study authors of newly identified studies by email, with 2 reminders over 1 month, if important study data or reporting appear to be missing or are unclear. When there are multiple publications of the same study, we will consider the earliest full publication of the primary outcome data to be the primary data source, while all others will be considered as secondary sources/associated publications. We will extract data from the primary source first, adding in data from the secondary source(s).

\section{Within-study risk of bias assessments}

For KQ1, to align with the previous review conduct [29], we will use the Cochrane Effective Practice and Organisation of Care (EPOC) Group's risk-of-bias tool [49]. Results by domain for all studies will be reported, although we will also code trials as being at low, moderate, or high risk of bias.
For KQ2, we will use the tool for preference-based studies as per GRADE guidance, which includes questions related to the choice/selection of representative participants; appropriate administration and choice of instrument; analysis and presentation of methods and results; instrument-described health state presentation, of all relevant outcomes and valid with respect to health state; patient understanding; and subgroup analysis to explore heterogeneity [23]. Critical appraisal tools from the Critical Appraisal Skills Programme [50] and the Centre for Evidence-Based Management [51] will be used for qualitative and cross-sectional/survey studies, respectively, in KQs 2 and 3.

For the trials included in the previous review [29], we will rely on the prior assessments by this team. For all other studies, two reviewers will independently assess the studies using the previous team's reviewer instructions and come to a consensus on the final scores for each question using a third reviewer where necessary. Each risk of bias tool will be piloted with a sample of at least five studies, using multiple rounds until agreement on all elements is high. These assessments will be incorporated into our assessment of the risk of bias across studies when assessing the certainty of the evidence for each outcome (see below).

\section{Preliminary grouping of intervention components (nodes)} Because there will be the possibility of many different combinations of interventions based on their components, we will form meaningful groups ("nodes" when referring to the NMA) before analysis. After the review team codes all study arms based on their intervention components (Table 2) and other key dimensions (e.g., recruitment setting, delivery personnel), but before any analysis, they will chart the data and consult with the Task Force and clinical experts to create and clarify decision rules for grouping interventions in a meaningful way. The primary consideration will be whether the interventions are considered a single component, multiple components, or multifactorial. Some single-component interventions, differing by single interventions but within the same intervention category in Table 2, may be grouped together (e.g., lighting and flooring). Groupings of different multicomponent and multifactorial interventions may focus on the number of studies to some extent, for example, home hazard assessment and modification combined with exercise may involve different types of exercise if few studies examine each type. Groupings will also focus on factors thought to relate to implementation, such as feasibility, acceptability, access, preferences of patients and providers, and/or modify effects. If requested by the Task Force, we will conduct one or more meta-regressions or stratified analyses using the pair-wise comparisons with UC to see where 
intervention effects may be modified based on a priori intervention covariates of interest including the inclusion of exercise (in multiple component interventions), dose, intensity, setting, and delivery provider. This would also potentially help prevent heterogeneity in the network meta-analysis. After this process, preliminary networks will be created and the synthesis started. In some cases, the final network configuration may be revised based on the assessment of the NMA, as described below.

\section{Data synthesis for KQ1 (benefits and harms)}

When a meta-analysis is not appropriate, a descriptive summary with accompanying tables and/or figures to present the data will be performed.

NMAs will be considered for all critical outcomes where indirect evidence exists for the outcome and connects to the network. This form of analysis simultaneously evaluates a suite of comparisons. A network of different comparisons is constructed, with nodes representing the different interventions, to consider both direct evidence from comparisons of interest (e.g., intervention B vs. UC) and indirect evidence from other comparisons where one intervention is in common, but not all (e.g., effects from intervention A vs. UC and from intervention A vs. B comparisons will contribute to the estimate of the "network" effect for intervention B vs. UC). For the important but not critical outcome of intervention-related AEs, and for comparisons with UC that are not included in an NMA based on intransitivity or other reasons, pairwise meta-analyses will be conducted where appropriate.

\section{Pairwise meta-analysis}

For pairwise meta-analysis, because of anticipated between-study heterogeneity, we will employ the DerSimonian Laird random-effects model using Stata. For dichotomous outcomes, we will report ORs or RaRs with corresponding 95\% CIs. For continuous outcomes, we will report a pooled mean difference using changes scores, when one measurement tool is used. We will use a standardized mean difference when combining two or more outcome scales measuring similar constructs based on clinical input. If suitable, we will transform the results back to the scale most frequently used. If we are not able to use a study's data in a meta-analysis (e.g., only p values are reported), we will comment on these findings and compare them with the results of the metaanalysis. Where SDs have been imputed or estimated we will perform sensitivity analysis by removing these studies. When event rates are less than 1\%, the Peto OR method will be used, unless control groups are of unequal sizes, large magnitude of the effect is observed, or when events become more frequent $(5-10 \%)$ where the
Mantel-Haenszel method without correction factor will be used [52]. The decision to pool studies will not be based on the statistical heterogeneity; the $I^{2}$ statistic will be reported but it is recognized that the $I^{2}$ is influenced by the number of studies and magnitude and direction of effects [52]. Rather, we will rely on interpretations of the clinical (related to our PICOTS) and methodological differences between studies. When heterogeneity in effects is seen, we will conduct subgroup or sensitivity analysis, using the same variables described in the section on assessment of transitivity in the NMAs. Effect estimates for each outcome will be transformed to risk differences to allow judgment of the clinical importance [53]. For outcomes having statistically significant effects, we will calculate the number needed to treat (NNT) and its $95 \%$ CI.

\section{Network meta-analysis}

We will employ random effects NMA and network meta-regressions in the most recent version of Stata available at the time of our analysis, using a frequentist approach that accounts for correlations between effect sizes from multi-arm studies [54]. The measure of treatment effect will be an OR with the exception of the rate of falls where we will report RaRs. The heterogeneity within the same treatment comparison will be measured with the tau-squared which represents the variance of the random effects distribution; this variance will be assumed to be common across the various treatment comparisons although sources of heterogeneity between different comparisons will be explored by network metaregressions during the assessment of intransitivity.

The assumptions underlying NMA are similar to standard pairwise meta-analysis, but there are additional issues of comparability that need to be considered to ensure the validity of results [55]. Indirect comparisons are not protected by randomization and may be confounded by differences between the trials.

\section{Assessment of transitivity}

Transitivity means that covariates that act as relative treatment effect modifiers are similar across different interventions, or adjusted for using meta-regression, so the effect of all treatments included in the model is generalizable across all included studies. Our exclusion criteria for certain populations expected to require different usual care, and for interventions provided in hospital and home-care settings are thought to prevent substantial intransitivity.

Across studies grouped by comparison, we will investigate the distribution of clinical and methodological covariates that, based on findings from other systematic reviews $[19,20,29]$, may be important effect modifiers related to the population or study design - age $(<80$ vs. 
$\geq 80$ years), previous fallers ( $100 \%$ vs. $>30 \leq 100 \%$ vs. general population risk of $\leq 30 \%$ ), recruited at hospitals, countries with the similar healthcare system to Canada (e.g., high-income, Organisation for Economic Cooperation and Development and predominantly universal health care [56]), and study design (i.e., follow-up after randomization $[<12$ vs. $\geq 12$ months]). We plan to use previous falls rather than increased/high-risk for falls based on various factors, because this is consistently shown to have a strong association with risk for falls (e.g., OR for any fall 2.8 [95\% confidence interval 2.4-3.3] and for recurrent fallers 3.5 [95\% CI 2.9-4.2]) $[57,58]$ and there is some evidence to suggest this risk factor alone may modify treatment effects [29].

We will use graphic methods, including weighting edges (lines between nodes) in the network plots based on covariates, to examine similarity between comparisons $[38,55]$.

Network meta-regressions will also be performed on the NMA to examine the influence of the aforementioned covariates; the change to the heterogeneity (tau value) will be tabulated. If one variable is thought to lead to important statistical heterogeneity, we may verify this with sensitivity analysis and, if necessary, either split the NMA into subgroups using the variables or adjust the NMA for the covariate. Otherwise, the results for relevant comparisons may be rated down for indirectness during the assessment of certainty (see below).

\section{Assessment of coherence}

Incoherence refers to differences between direct and various indirect effect estimates that contribute to the overall "network" estimate for each comparison. We will assess incoherence both locally (per comparison) using the Separate Indirect from Direct Evidence (SIDE, or node-splitting approach [59]) and globally (all treatment effects and all possible inconsistency factors are considered simultaneously) using the design-by-treatment interaction model [54] and comparison of the consistency model to the inconsistency model. These methods provide $p$ values, and $<0.01$ and $<0.10$ may be considered to indicate major and some incoherence [60]. Major global incoherence may result in reconfiguration of the network or not conducting the NMA; otherwise, the degree of incoherence will be considered during the assessment of the certainty of effects as described in that section.

\section{Presentation of results}

We will present all final network plots, with the size of the nodes corresponding to the number of participants randomized to each treatment and the lines/edges weighted by the number of trials evaluating the comparison. The summary ORs or RaRs and 95\% CIs for all pairwise comparisons will be presented in a league table (including all direct [where available] and network estimates). To rank the various treatments for each outcome relative to $\mathrm{UC}$, we will use surface under the cumulative ranking curves (SUCRA) and present the SUCRA values in ranking plots; if useful for the working group, we will also create a heat rank plot to display the SUCRA values for all outcomes analyzed. SUCRA values account both for the location and the variance (uncertainty/imprecision) of all relative treatment effects [38]. For each NMA, the overall risk for the UC group will be calculated using the variance-stabilizing Freeman-Tukey double arcsine approach. Network estimates for each node compared with usual care will be transformed to risk differences to allow judgment of the clinical importance [53]. For outcomes having statistically significant effects, we will calculate the number needed to treat (NNT).

\section{Small study effects}

For the NMA outcomes, we will consider using comparison-adjusted funnel plots to assess for small study bias, if clinical input suggests there is rationale for a particular characteristic to be associated with small study effects, and assumptions about the direction of small studies can be made (i.e., treatments need to be ordered in a meaningful way) [38]. Otherwise, we will conduct a funnel-plot grouping all interventions versus usual care, and if bias is evident, we will then assess individual interventions versus $\mathrm{UC}$ (if $\geq 10 \mathrm{RCTs}$ ) and assess for this bias as usual for pairwise comparisons.

For outcomes where pairwise meta-analysis is used and when 10 or more RCTs are in the comparison, we will analyze for small-study effects both visually using the funnel plot and quantitatively using Egger's test [61] (continuous outcomes) or Harbord's test [62] (dichotomous outcomes).

\section{Data synthesis for KQs 2 and 3 (values and preferences)}

This analysis will be guided by a narrative synthesis approach [63]. We will likely rely on textual descriptions and groupings/clusterings to develop a preliminary synthesis of the findings. We will explore relationships between the data by comparing and contrasting study findings while considering study methodology (e.g., timing of outcome measurement), populations (e.g., age, experience with the outcome or intervention type), outcome presentations provided to participants (relevant only for KQ2), comparisons (between outcomes in KQ2 and between differing intervention attributes in KQ3), and analytical approaches. Groupings based on key differences between studies will be created; for example, KQ2 findings from utility and non-utility studies will be separated. Within-study subgroup analyses will be 
interpreted. We do not anticipate performing metaanalysis, although this may be possible for utility values for some health states/outcomes such as hip fractures if there are two or more studies using the same measurement method in similar populations. If undertaken, we will use a random-effects model. Results for health-state utilities will be separated by utility measurement tool (e.g., EQ-5D, time trade-off) and the main covariates of interest for subgroup analysis will be age, sex, time since fracture ( $\leq 12$ months vs. $>12$ months), and fracture history [64].

\section{Assessing the certainty of the evidence}

We will assess the certainty of evidence for all outcomes, for the effects of each intervention grouping versus UC. For outcomes analyzed by pairwise meta-analysis or no meta-analysis, we will follow current GRADE guidance [23, 34, 65-67]. For findings from NMA, we will be guided by the CINeMa approach and use CINeMA software for some assessments, which is based on the GRADE framework, although has conceptual and semantic differences [68]. The assessment covers six domains: within-study bias, across-studies bias (i.e., publication and other reporting biases), indirectness, imprecision, heterogeneity (i.e., variation between studies within a comparison), and incoherence (i.e., variation between direct and indirect sources of evidence across comparisons). Findings during the assessment of transitivity and incoherence of the NMA network will contribute information to support certainty ratings for indirectness and incoherence, respectively, as described further below. Similar to GRADE, judgments for each domain are of no concern, some concern, or major concerns, and for each outcome are of very low, low, moderate, or high. Some of the assessments rely on a percentage contribution matrix (see below). Each outcome starts at high certainty and is rated down for concerns. The six CINeMA domains are interconnected and should be considered jointly rather than in isolation [68]; if two concerns are highly related, we will not rate down twice.

\section{Percentage contribution matrix}

Most studies in a network contribute some indirect information to every estimate of a relative treatment effect. Studies contribute more when their results are precise (e.g., large studies), when they provide direct evidence or when the indirect evidence does not involve many "steps." The contribution made by each study can be quantified to each relative treatment effect on a 0 to $100 \%$ scale. These quantities can be presented in a percentage contribution matrix.

\section{Within-study risk of bias}

CINeMA combines the studies' percent contributions with the risk of bias judgments to evaluate study limitation for each estimate of a relative treatment effect from a network meta-analysis. More concern about study limitations exists when there is a larger contribution from studies at high or moderate risk of bias. With the percentage contributions, weighted average levels of overall risk of bias are produced. Scores of $-1,0$, and 1 are considered low, moderate, and high risk of bias.

\section{Across-studies bias}

The CINeMA approach provides conditions that would be considered to provide judgments about "suspected" or "undetected" bias. Suspected bias entails (i) failure to include unpublished data, (ii) meta-analysis is based on a small number of positive "early" studies, (iii) the comparison has been funded primarily by industry-funded trials, or (iv) there is existing evidence of reporting bias. A judgment of undetected bias arises from (i) inclusion of unpublished studies with similar findings to those published, (ii) protocols and clinical trial registries are available for many trials and important discrepancies are not found, and (iii) the effects from small studies do not differ from those from large studies [68]. Although our inclusion of gray literature and many studies, as well as the non-pharmacologic topic, would suggest no suspicion of bias, we expect [29] a large portion of the studies to have concerns about selective reporting (e.g., missing outcomes). Outcomes may be rated down if there is evidence of small-study effects or if several studies in the review did not report on the outcome despite inclusion in their protocol and/or when clinical input suggests it should have measured. This approach is very similar to that used for pair-wise meta-analysis.

\section{Indirectness}

Our inclusion and exclusion criteria are fairly rigid and are expected to capture studies of high relevance to the Task Force's main population, outcomes, and settings of interest. Nevertheless, some comparisons may have some indirectness. Each study included in the review will be coded based on its overall relevance to the main PICOTS (low, moderate, high). Similar to the approach for within-study risk of bias, the findings will then be combined with the percentage contribution of the studies to each comparison to provide a value weighted by each comparison. We will also consider information provided in our assessment of transitivity, when we weighted the edges in the network plots based on covariates in the associated studies to examine similarity between comparisons. If the edges for the comparison are of similar width to those in the majority of comparisons in the network, we will be less concerned about indirectness. 


\section{Imprecision}

Imprecision will be assessed in a similar manner as for findings from pair-wise meta-analysis [69]. Because this review is not focusing on the difference in effects between all of the different comparisons, determining a range of equivalence for comparing different interventions (e.g., how much better one needs to be than another) will not be conducted. We will rate down the evidence for imprecision, if, using the network estimate, (i) the effect could be considered clinically important based on the network "point" estimate (e.g., OR $\leq 0.8$ for reducing fallers) but the $95 \% \mathrm{CI}$ crosses the null or (ii) the estimate is likely too small to be important (e.g., OR 0.95 ) but the $95 \%$ CI includes values indicating the possibility of an important effect in either direction. Rating down by two levels may occur if the effect appears to be of little to no difference but the $95 \%$ CI is very wide, indicating possible benefit and harm (e.g., spanning ORs of both $<0.75$ and $>1.25$ ) [69].

\section{Heterogeneity}

The concordance between assessments based on confidence intervals, which do not capture heterogeneity, and prediction intervals, showing where the true effect of a new study similar to the existing studies is expected to lie, can be used to assess the importance of heterogeneity. The effect of the heterogeneity on the conclusions will be considered (see imprecision for general rules on effect sizes), and if the predictive intervals do not add any concern over that already assessed for imprecision, we will not rate down for this domain. Predictive intervals derived from meta-analyses with very few studies can be unreliable and this will be taken into account.

\section{Incoherence}

We will use results from our local (per comparison; using SIDE, or node-splitting approach [59]) and global [54] assessments of incoherence. Both methods provide $p$ values, and we will consider $<0.01$ and $<0.10$ to indicate major and some incoherence [60]. Comparisons that have $>90 \%$ direct evidence will not be rated down. For comparisons that have only indirect evidence (i.e., local coherence not relevant), we will rate down due to incoherence one or two levels depending on whether the $p$ value of the design by treatment interaction model was between 0.01 and 0.10 or less than 0.01 , respectively. If there is $>0 \%$ and $<90 \%$ direct evidence, we will base the decision on the more relevant method (e.g., high reliance on node splitting when more direct evidence). We will also consider the $95 \%$ CIs from the direct and indirect evidence for each comparison; if both are showing the same direction of effect, but differing magnitudes of beneficial effects, we will have less concern.
Input from the Task Force will be used when the review team conducts the certainty assessments for each outcome, for example, when appraising the applicability/ indirectness of the studies in terms of the population of interest to their recommendation.

\section{Discussion}

The review will be published in an open-access journal and reported using standard checklists for systematic reviews and network meta-analysis [70, 71]. The results section of the review will include a description of the flow of literature and characteristics of all studies, results of all analyses, including planned subgroup and sensitivity analyses as well as the assessment of the NMAs, and summary of finding tables incorporating assessment of our confidence in the estimates of effect. In the discussion, we will summarize the main findings and their implications, compare our findings to others, and discuss the limitations of the review and the available literature. The results will be used by the Task Force for developing recommendations about fall prevention in community-dwelling older adults. It will also serve as a comprehensive review for clinicians and other decision makers on the effects of interventions and relevant patient preferences.

\section{Protocol amendments}

We will report on any deviations from the protocol within the final manuscript.

\section{Supplementary Information}

The online version contains supplementary material available at https://doi. org/10.1186/s13643-020-01572-7.

Additional file 1.

\section{Abbreviations}

ADLs: Activities of daily living; Cl: Confidence interval; EPOC: Cochrane Effective Practice and Organisation of Care; GRADE: Grading of Recommendations Assessment, Development and Evaluation; ICC: Intraclass correlation coefficient; KQ: Key question; NMA: Network meta-analysis; OR: Odds ratio; PHAC: Public Health Agency of Canada; PICOTS: Population, interventions, comparators, outcomes, timing, setting; RaR: Rate ratio; RCT: Randomized controlled trial; RR: Relative risk; SD: Standard deviation; SIDE: Separate indirect from direct evidence; SUCRA: Surface under the cumulative ranking; UC: Usual care

\section{Acknowledgements}

We would like to acknowledge the Canadian Task Force on Preventive Health Care members who are not in the working group on this topic (Ahmed M. Abou-Setta, Roland Grad, Donna Reynolds, Guylène Thériault, Michael Kidd, Tina Korownyk, Scott Klarenbach, John Leblanc, Nav Persaud). We also thank Ms. Tara Landy, MLIS, for peer-reviewing the search strategies.

\section{Authors' contributions}

JP drafted the majority of this manuscript. DK-L developed the search strategy for KQ2. BV provided input for the sections on data extraction and analysis and reviewed the manuscript. JJR, HC, EL, AEM, BDT, BJW, AT, CD, $\mathrm{ME}, \mathrm{JH}-\mathrm{L}$, and $\mathrm{JM}$ provided clinical/content input, assisted with developing the inclusion and exclusion criteria for the review, and provided input to all 
sections of the protocol. LAT, BM, and ERH provided methodological input related to guideline development during the development of the protocol and reviewed the manuscript. LT drafted some of the background. ACT, SES, and SMT contributed to the development of the methods and reviewed the manuscript. The authors read and approved the final version of the manuscript.

\section{Funding}

This protocol and the subsequent review will be conducted for the Public Health Agency of Canada; however, it does not necessarily represent the views of the Government of Canada. The Staff of the Global Health and Guidelines Division at the Public Health Agency of Canada (LAT, BM, ERH) provided input during the development of this protocol and reviewed the protocol, but will not be taking part in the selection of studies, data extraction, analysis, or interpretation of the findings. ACT is funded by a Tie 2 Canada Research Chair in Knowledge Synthesis; SES is funded by a Tier 1 Canada Research Chair in Knowledge Translation, and LH is funded by a Tier 1 Canada Research Chair in Knowledge Synthesis and Translation.

\section{Availability of data and materials}

Not applicable.

\section{Ethics approval and consent to participate}

Not applicable

\section{Consent for publication}

Not applicable

\section{Competing interests}

All authors declare that they have no competing interests.

\section{Author details}

${ }^{1}$ Alberta Research Centre for Health Evidence, University of Alberta, 1140587 Avenue, Edmonton, AB T6G 1C9, Canada. ${ }^{2}$ Department of Family Medicine, McMaster University, Hamilton, Canada. ${ }^{3} \mathrm{Global}$ Health and Guidelines Division, Public Health Agency of Canada, Ottawa, Canada. ${ }^{4}$ Occupational Science and Occupational Therapy Department, University of Toronto, Toronto, Canada. ${ }^{5}$ Cumming School of Medicine, University of Calgary and Alberta Health Services, Calgary, Canada. ${ }^{6}$ Faculty of Medicine, McGill University, Montreal, Canada. ${ }^{7}$ Community Health and Humanities, Faculty of Medicine, Memorial University of Newfoundland, St. John's, Canada. ${ }^{8}$ Memorial University of Newfoundland, St. John's, Canada. ${ }^{9}$ School of Rehabilitation Therapy, Queen's University, Kingston, Canada. ${ }^{10}$ Department of Family Medicine and Emergency Medicine, Université Laval, Quebec City, Canada. ${ }^{11}$ Department of Medicine, University of Calgary, Calgary, Canada.

${ }^{12}$ Schlegel Specialist in Mobility and Falls, Schlegel-UW Research Institute for Aging, Department of Family Medicine, McMaster University, Hamilton, Canada. ${ }^{13}$ Knowledge Translation Program, Li Ka Shing Knowledge Institute, St Michael's Hospital, Unity Health Toronto, Toronto, Canada.

\section{Received: 21 February 2020 Accepted: 23 December 2020}

\section{Published online: 09 January 2021}

\section{References}

1. Public Health Agency of Canada. Seniors' falls in Canada: second report. Ottawa: Public Health Agency of Canada; 2014. Available from: https://www. canada.ca/en/public-health/services/health-promotion/aging-seniors/ publications/publications-general-public/seniors-falls-canada-second-report. html. Accessed 03 Sept 2020

2. Berry SD, Miller RR. Falls: epidemiology, pathophysiology, and relationship to fracture. Curr Osteoporo Reports. 2008;6:149-54.

3. Tinetti ME, Doucette J, Claus E, Marottoli R. Risk factors for serious injury during falls by older persons in the community. J Am Geriatr Soc. 1995;43: 1214-21.

4. Scuffham P, Chaplin S, Legood R. Incidence and costs of unintentional falls in older people in the United Kingdom. J Epidemiol Community Health. 2003:57:740-4

5. Burns ER, Stevens JA, Lee R. The direct costs of fatal and non-fatal falls among older adults - United States. J Safety Res. 2016;58:99-103.

6. Scott V, Wagar L, Elliott S. Falls and related injuries among older Canadians: fall-related hospitalizations \& intervention initiatives. Prepared on behalf of the Public Health Agency of Canada Division of Aging and Seniors. Victoria: Victoria Scott Consulting; 2010. See page 8 in http://www.hiphealth.ca/ media/research_cemfia_phac_epi_and_inventor_20100610.pdf. Accessed 03 Sept 2020.

7. Jorstad EC, Hauer K, Becker C, Lamb SE. Measuring the psychological outcomes of falling: a systematic review. J Am Geriatr Soc. 2005;53:501-10.

8. Stenhagen $\mathrm{M}$, Ekstrom $\mathrm{H}$, Nordell $\mathrm{E}$, Elmstahl S. Accidental falls, healthrelated quality of life and life satisfaction: a prospective study of the general elderly population. Arch Gerontol Geriatr. 2014;58:95-100.

9. Taylor-Piliae RE, Peterson R, Mohler MJ. Clinical and community strategies to prevent falls and fall-related injuries among community-dwelling older adults. Nurs Clin North Am. 2017;52:489-97.

10. Girgis CM, Clifton-Bligh RJ, Hamrick MW, Holick MF, Gunton JE. The roles of vitamin D in skeletal muscle: form, function, and metabolism. Endocr Rev. 2013:34:33-83.

11. Gunton JE, Girgis CM. Vitamin D and muscle. Bone Rep. 2018:8:163-7.

12. Yardley L, Beyer N, Hauer K, Kempen G, Piot-Ziegler C, Todd C. Development and initial validation of the Falls Efficacy Scale-International (FES-I). Age Ageing. 2005:34:614-9.

13. Rikli RE, Jones CJ. Development and validation of criterion-referenced clinically relevant fitness standards for maintaining physical independence in later years. Gerontologist. 2013;53:255-67.

14. Berg KO, Maki BE, Williams JI, Holliday PJ, Wood-Dauphinee SL. Clinical and laboratory measures of postural balance in an elderly population. Arch Phys Med Rehabil. 1992;73:1073-80.

15. Podsiadlo D, Richardson S. The timed "Up \& Go": a test of basic functional mobility for frail elderly persons. J Am Geriatr Soc. 1991;39:142-8.

16. Park SH. Tools for assessing fall risk in the elderly: a systematic review and meta-analysis. Aging Clin Exp Res. 2018;30:1-16

17. Lusardi MM, Fritz S, Middleton A, Allison L, Wingood M, Phillips E, et al. Determining risk of falls in community dwelling older adults: a systematic review and meta-analysis using posttest probability. J Geriatr Phys Ther. 2017:40:1-36

18. Guirguis-Blake JM, Michael YL, Perdue LA, Coppola EL, Beil TL. Interventions to prevent falls in older adults: updated evidence report and systematic review for the US Preventive Services Task Force. JAMA. 2018:319:1705-16.

19. Hopewell SA, Adedire O, Copsey BJ, Boniface GJ, Sherrington C, Clemson L, et al. Multifactorial and multiple component interventions for preventing falls in older people living in the community. Cochrane Database Syst Rev. 2018;7(7):CD012221.

20. Sherrington CF, Fairhall NJ, Wallbank GK, Tiedemann A, Michaleff ZA, Howard $\mathrm{K}$, et al. Exercise for preventing falls in older people living in the community. Cochrane Database Syst Rev. 2019;1(1):CD012424.

21. Lamb SE, Becker C, Gillespie LD, Smith JL, Finnegan S, Potter R, et al. Reporting of complex interventions in clinical trials: development of a taxonomy to classify and describe fall-prevention interventions. Trials. 2011; $12: 125$

22. Atun $\mathrm{R}$. What are the advantages and disadvantages of restructuring a health care system to be more focused on primary care services? Copenhagen: WHO Regional Office for Europe; 2004. http://www.euro.who. int/document/e82997.pdf. Accessed 03 Sept 2020.

23. Zhang Y, Alonso-Coello P, Guyatt GH, Yepes-Nunez JJ, Akl EA, Hazlewood G, et al. GRADE Guidelines: 19. Assessing the certainty of evidence in the importance of outcomes or values and preferences-Risk of bias and indirectness. J Clin Epidemiol. 2019:111:94-104.

24. Nyman SR, Victor CR. Older people's participation in and engagement with falls prevention interventions in community settings: an augment to the Cochrane systematic review. Age Ageing. 2012;41:16-23.

25. Yardley L, Kirby S, Ben-Shlomo Y, Gilbert R, Whitehead S, Todd C. How likely are older people to take up different falls prevention activities? Prev Med. 2008:47:554-8

26. Franco MR, Howard K, Sherrington C, Ferreira PH, Rose J, Gomes JL, et al. Eliciting older people's preferences for exercise programs: a best-worst scaling choice experiment. J Physiother. 2015:61:34-41.

27. Dorresteijn TA, Rixt Zijlstra GA, Van Eijs YJ, Vlaeyen JW, Kempen Gl. Older people's preferences regarding programme formats for managing concerns about falls. Age Ageing. 2012;41:474-81

28. Zhang Y, Coello PA, Brozek J, Wiercioch W, Etxeandia-Ikobaltzeta I, AkI EA, et al. Using patient values and preferences to inform the importance of health outcomes in practice guideline development following the GRADE approach. Health Qual Life Outcomes. 2017;15:52. 
29. Tricco AC, Thomas SM, Veroniki AA, Hamid JS, Cogo E, Strifler L, et al. Comparisons of interventions for preventing falls in older adults: a systematic review and meta-analysis. JAMA. 2017;318:1687-99.

30. Cheng PT, Tan L, Ning P, Li L, Gao Y, Wu Y, et al. Comparative effectiveness of published interventions for elderly fall prevention: a systematic review and network meta-analysis. Int J Environ Res Public Health. 2018:15:498.

31. Rimland JM, Abraha I, Dell'Aquila G, Cruz-Jentoft A, Soiza R, Gudmusson A, et al. Effectiveness of non-pharmacological interventions to prevent falls in older people: a systematic overview. The SENATOR Project ONTOP Series. PLoS One. 2016;11:e0161579.

32. Canadian Task Force on Preventive Health Care. Procedure Manual. 2014. https://canadiantaskforce.ca/methods/. Accessed 03 Sept 2020.

33. Higgins JPT, Thomas J, Chandler J, Cumpston M, Li T, Page MJ, et al. (Eds). Cochrane handbook for systematic reviews of interventions, version 6.0. 2019. https://training.cochrane.org/handbook. Accessed 03 Sept 2020.

34. Zhang Y, Coello PA, Guyatt GH, Yepes-Nunez JJ, Akl EA, Hazlewood G, et al. GRADE guidelines: 20. Assessing the certainty of evidence in the importance of outcomes or values and preferences-inconsistency, imprecision, and other domains. J Clin Epidemiol. 2019;111:83-93.

35. Schunemann H, Brozek J, Guyatt G, Oxman A (Eds): GRADE Handbook. 2013. http://gdt.guidelinedevelopment.org/app/handbook/handbook.html. Accessed 03 Sept 2020.

36. Moher D, Shamseer L, Clarke M, Ghersi D, Liberati A, Petticrew M, et al. Preferred reporting items for systematic review and meta-analysis protocols (PRISMA-P) 2015 statement. Syst Rev. 2015;4:1.

37. Bucher HC, Guyatt GH, Griffith LE, Walter SD. The results of direct and indirect treatment comparisons in meta-analysis of randomized controlled trials. J Clin Epidemiol. 1997;50:683-91.

38. Chaimani A, Higgins JP, Mavridis D, Spyridonos P, Salanti G. Graphical tools for network meta-analysis in STATA. PLoS One. 2013;8:e76654.

39. Salanti G. Indirect and mixed-treatment comparison, network, or multipletreatments meta-analysis: many names, many benefits, many concerns for the next generation evidence synthesis tool. Res Synth Methods. 2012;3:8097.

40. Moher D, Pham B, Klassen TP, Schulz KF, Berlin JA, Jadad AR, et al. What contributions do languages other than English make on the results of meta-analyses? J Clin Epidemiol. 2000;53:964-72.

41. Morrison A, Polisena J, Husereau D, Moulton K, Clark M, Fiander M, et al. The effect of English-language restriction on systematic review-based metaanalyses: a systematic review of empirical studies. Int J Technol Assess Health Care. 2012;28:138-44.

42. McGowan J, Sampson M, Salzwedel DM, Cogo E, Foerster V, Lefebvre C. PRESS peer review of electronic search strategies: 2015 guideline statement. J Clin Epidemiol. 2016;75:40-6.

43. Liberati A, Altman DG, Tetzlaff J, Mulrow C, Gotzsche PC, loannidis JP, et al The PRISMA statement for reporting systematic reviews and meta-analyses of studies that evaluate health care interventions: explanation and elaboration. J Clin Epidemiol. 2009;62:e1-34.

44. O'Neill J, Tabish H, Welch V, Petticrew M, Pottie K, Clarke M, et al. Applying an equity lens to interventions: using PROGRESS ensures consideration of socially stratifying factors to illuminate inequities in health. J Clin Epidemiol. 2014;67:56-64.

45. Lamb SE, Jorstad-Stein EC, Hauer K, Becker C. Development of a common outcome data set for fall injury prevention trials: the Prevention of Falls Network Europe consensus. J Am Geriatr Soc. 2005:53:1618-22.

46. Schwenk M, Lauenroth A, Stock C, Moreno RR, Oster P, McHugh G, et al. Definitions and methods of measuring and reporting on injurious falls in randomised controlled fall prevention trials: a systematic review. BMC Med Res Methodol. 2012;12:50.

47. Follmann D, Elliott P, Suh I, Cutler J. Variance imputation for overviews of clinical trials with continuous response. J Clin Epidemiol. 1992;45:769-73.

48. Smeeth L, Ng ES. Intraclass correlation coefficients for cluster randomized trials in primary care: data from the MRC Trial of the Assessment and Management of Older People in the Community. Control Clin Trials. 2002; 23:409-21.

49. Cochrane Effective Practice and Organisation of Care (EPOC). Suggested risk of bias criteria for EPOC reviews. 2017. https://epoc.cochrane.org/sites/epoc. cochrane.org/files/public/uploads/Resources-for-authors2017/suggested_ risk_of_bias_criteria_for_epoc_reviews.pdf. Accessed 29 Sept 2020.

50. Critical Appraisal Skills Programme. CASP qualitative checklist. Oxford: Middleway; 2018. https://casp-uk.net/casp-tools-checklists/. Accessed 03 Sept 2020.
51. Centre for Evidence-Based Management (CEBMa). Appraisal of a crosssectional study (survey). Leiden; 2018. https://www.cebma.org/resourcesand-tools/what-is-critical-appraisal/. Accessed 03 Sept 2020.

52. Fu R, Gartlehner G, Grant M, Shamliyan T, Sedrakyan A, Wilt TJ, et al. Conducting quantitative synthesis when comparing medical interventions: AHRQ and the effective health care program. J Clin Epidemiol. 2011;64:1187-97.

53. Murad MH, Montori VM, Walter SD, Guyatt GH. Estimating risk difference from relative association measures in meta-analysis can infrequently pose interpretational challenges. J Clin Epidemiol. 2009;62:865-7.

54. White IR, Barrett JK, Jackson D, Higgins JP. Consistency and inconsistency in network meta-analysis: model estimation using multivariate metaregression. Res Synth Methods. 2012;3:111-25.

55. Donegan S, Williamson P, D'Alessandro U, Tudur SC. Assessing key assumptions of network meta-analysis: a review of methods. Res Synth Methods. 2013;4:291-323.

56. Barua B, Jacques D. Comparing performance of universal health care countries, 2018: Fraser Institute; 2018. https://www.fraserinstitute.org/sites/ default/files/comparing-performance-of-universal-health-care-countries-2018. pdf. Accessed 03 Sept 2020.

57. Deandrea S, Lucenteforte E, Bravi F, Foschi R, La Vecchia C, Negri E. Risk factors for falls in community-dwelling older people: a systematic review and meta-analysis. Epidemiology. 2010;21:658-68.

58. Ambrose AF, Paul G, Hausdorff JM. Risk factors for falls among older adults: a review of the literature. Maturitas. 2013;75:51-61.

59. Dias S, Welton NJ, Caldwell DM, Ades AE. Checking consistency in mixed treatment comparison meta-analysis. Stat Med. 2010;29:932-44.

60. Huhn M, Nikolakopoulou A, Schneider-Thoma J, Krause M, Samara M, Peter $\mathrm{N}$, et al. Comparative efficacy and tolerability of 32 oral antipsychotics for the acute treatment of adults with multi-episode schizophrenia: a systematic review and network meta-analysis. Lancet. 2019;394:939-51.

61. Egger M, Davey Smith G, Schneider M, Minder C. Bias in meta-analysis detected by a simple, graphical test. BMJ. 1997;315:629-34.

62. Harbord RM, Egger M, Sterne JA. A modified test for small-study effects in meta-analyses of controlled trials with binary endpoints. Stat Med. 2006;25: 3443-57.

63. Popay J, Roberts H, Sowden A, Petticrew M, Arai L, Rodgers M, et al. Guidance on the conduct of narrative synthesis in systematic reviews: a product from the ESRC Methods Programme. 2006. http://citeseerx.ist.psu.edu/viewdoc/ download?doi=10.1.1.178.3100\&rep=rep1\&type=pdf. Accessed 03 Sept 2020.

64. Si L, Winzenberg TM, de Graaff B, Palmer AJ. A systematic review and metaanalysis of utility-based quality of life for osteoporosis-related conditions. Osteoporos Int. 2014:25:1987-97.

65. Guyatt GH, Oxman AD, Schunemann HJ, Tugwell P, Knottnerus A. GRADE guidelines: a new series of articles in the Journal of Clinical Epidemiology. J Clin Epidemiol. 2011;64:380-2.

66. Hultcrantz M, Rind D, Akl EA, Treweek S, Mustafa RA, lorio A, et al. The GRADE Working Group clarifies the construct of certainty of evidence. J Clin Epidemiol. 2017:87:4-13.

67. Murad MH, Mustafa RA, Schunemann HJ, Sultan S, Santesso N. Rating the certainty in evidence in the absence of a single estimate of effect. Evid Based Med. 2017;22:85-7.

68. Nikolakopoulou A, Higgins J, Papakonstantinou T, Chaimani A, Del Giovane $\mathrm{C}$, Egger $\mathrm{M}$, et al. Assessing confidence in the results of network metaanalysis (CINeMA). 2019. https://www.biorxiv.org/content/biorxiv/early/2019/ 04/05/597047.full.pdf. Accessed 03 Sept 2020.

69. Guyatt GH, Oxman AD, Kunz R, Brozek J, Alonso-Coello P, Rind D, et al. GRADE guidelines 6. Rating the quality of evidence--imprecision. J Clin Epidemiol. 2011;64:1283-93.

70. Hutton B, Salanti G, Caldwell DM, Chaimani A, Schmid CH, Cameron C, et al. The PRISMA extension statement for reporting of systematic reviews incorporating network meta-analyses of health care interventions: checklist and explanations. Ann Intern Med. 2015;162:777-84.

71. Moher D, Liberati A, Tetzlaff J, Altman DG. Preferred reporting items for systematic reviews and meta-analyses: the PRISMA statement. BMJ. 2009; $339:$ b2535.

\section{Publisher's Note}

Springer Nature remains neutral with regard to jurisdictional claims in published maps and institutional affiliations. 